\title{
DESIGN AND COLD MODEL TEST OF KOMAC CCDTL*
}

\author{
Y.S. Cho", J.M. Han, H.E Ahn, B.H. Choi \\ Korea Atomic Energy Research Institute, P.O.Box 105, Taejon, Korea 305-600
}

\begin{abstract}
For the first phase of the KOMAC (Korea Multi-purpose Accelerator Complex) project, a CCDTL (Coupled Cavity Drift Tube Linac) which accelerates a $20 \mathrm{~mA} \mathrm{cw}$ proton beam from $3 \mathrm{MeV}$ to $20 \mathrm{MeV}$ is designed. An Al cold model is fabricated to check the design, the tuning method, and the coupling coefficients. To check the engineering design, a $\mathrm{Cu}$ cold model is fabricated. The design and the test results will be presented.
\end{abstract}

\section{INTRODUCTION}

The KOMAC CCDTL has been designed to accelerate a $20 \mathrm{~mA} \mathrm{cw}$ proton/H- beam from a $350 \mathrm{MHz}, 3 \mathrm{MeV} \mathrm{cw}$ RFQ and to inject the $100 \mathrm{MeV}$ beam to a $700 \mathrm{MHz}$, final energy $1 \mathrm{GeV}$ cw superconducting linac. The CCDTL is a coupled cavity drift tube linac (CCDTL) structure $[1,2]$ which allows the focusing magnets to remain outside the vacuum system and does not require permanent magnets that are susceptible to radiation damage due to the high average beam current. The CCDTL structure is less efficient than a conventional DTL, but relatively easier to fabricate and operate.

\section{KOMAC CCDTL DESIGN}

\subsection{RF Structure}

The $700 \mathrm{MHz}$ frequency, which is the same frequency of the superconducting linac, can be used for the CCDTL due to the ample space for the quadrupoles. The design parameters of the CCDTL cavity are shown in Table 1. These values, except for the aperture, are conservative for fabrication and $\mathrm{cw}$ operation.

Table 1: Design Parameters of the CCDTL cavity

- Frequency : $700 \mathrm{MHz}$

- Space for Quadrupole : $>8 \mathrm{~cm}$

- Real Estate E : $<1 \mathrm{MV} / \mathrm{m}$

- Surface E : <0.9 Kilpatrick

- Synchronous Phase : -60 -30 degree

- Focusing : $8 \beta \lambda$ FODO

- Aperture : Acceptance $>2$ transverse emit.

*Work supported by the Korea Ministry of Science and Technology

\#Email: choys@nanum.kaeri.re.kr
The aperture of the CCDTL can be determined by iterative calculations of the shunt impedance and the beam trajectory for the optimisation. A larger aperture decreases the shunt impedance, but increases the ratio of aperture to beam size (less beam loss). The optimised aperture of the CCDTL is shown in Fig. 1. The transition energy for the number of gaps per focusing period is determined by the space for the quadrupole magnet.

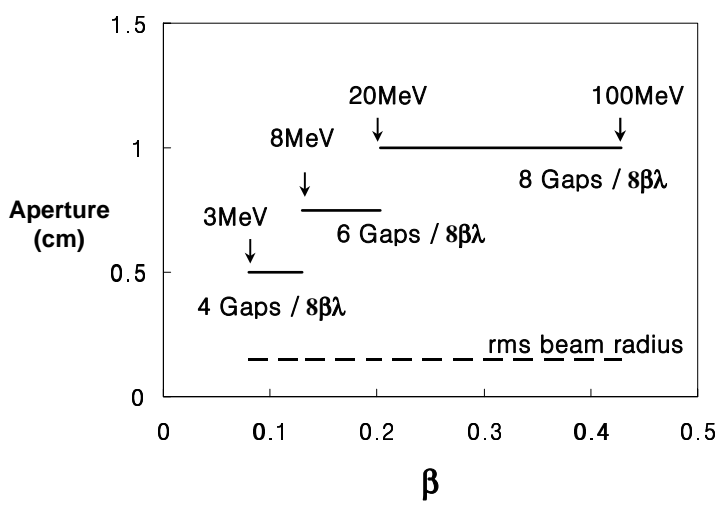

Figure 1: Aperture of the CCDTL and Beam Size

The cavity shapes are determined by SUPERFISH code. Fig. 2 shows the plot of the effective shunt impedance for the cavity and the real estate effective shunt impedance versus particle velocity. In spite of the small aperture, the effective shunt impedance is small in the first part of the CCDTL. This is not serious problem because this part works as a buncher and a matching section.

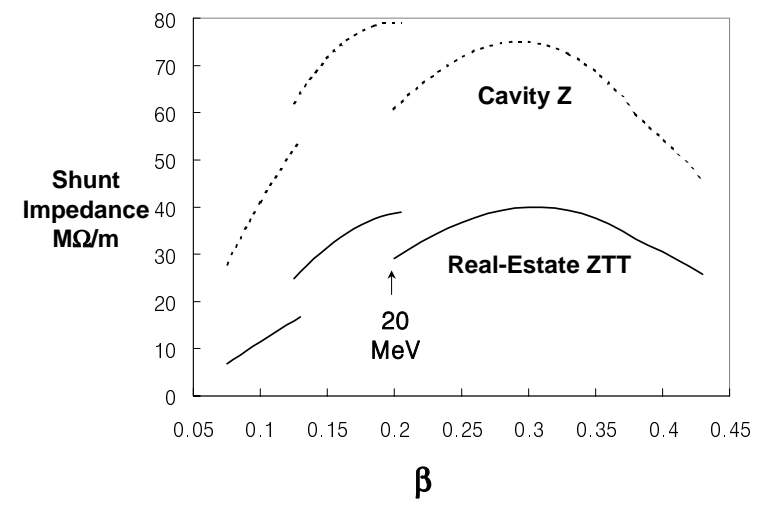

Figure 2: Shunt Impedance versus particle velocity for the CCDTL Cavity 


\subsection{Beam Dynamics}

Beam dynamics for the KOMAC CCDTL are performed using the PARMILA code. For longitudinal matching with $350 \mathrm{MHz}$ RFQ, the synchronous phases and the amplitudes of the first part of the CCDTL are adjusted [3].

The beam trajectory and the emittance in the CCDTL are shown in Fig. 3 and 4 with the beam from the RFQ (longitudinal emittance: 0.4 pi degree $\mathrm{MeV}$, transverse rms emittance: 0.32 pi $\mathrm{mm}$ mrad) with 100,000 particles. The GL of EMQ is $2.6 \mathrm{~T}$, and the length of poles is $6 \mathrm{~cm}$. The phase and amplitude of the first part for matching is given to obtain the smallest emittance growth. In the final simulation, there is virtually no growth in transverse emittance, and no more than $20 \%$ growth in longitudinal emittance, which is not critical.

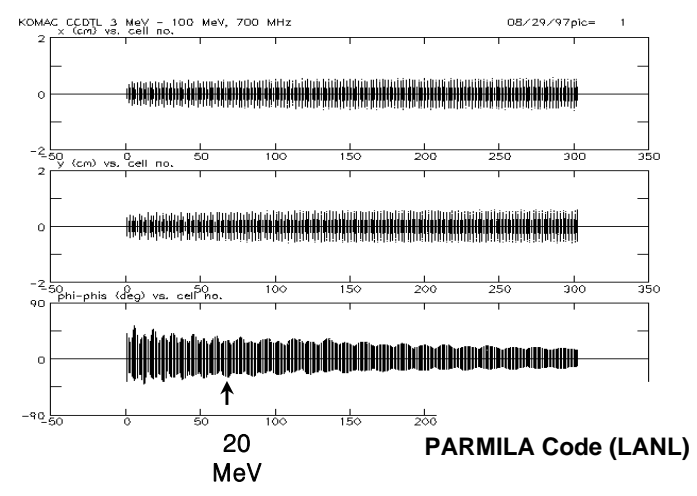

Figure 3: Beam trajectory in the CCDTL

To estimate the tolerances of the CCDTL structure, an error analysis has been done with PARMILA code. With the error in Table 2, which is achievable, the beam envelop calculated with PARMILA code does not grow more than 20\%, as shown in Fig. 5.

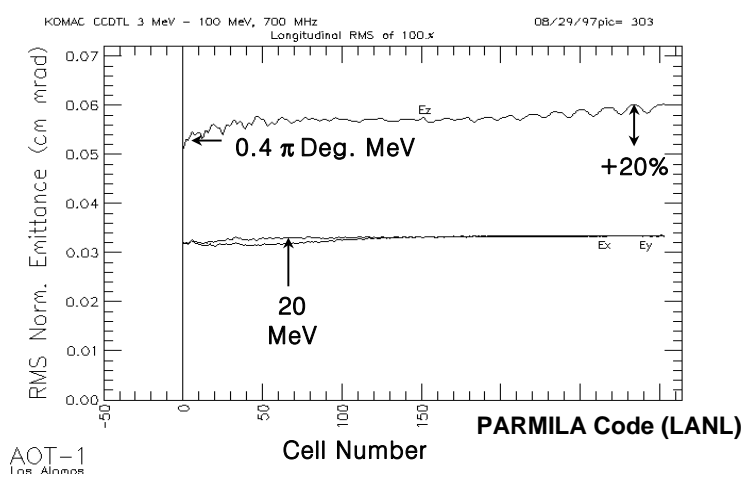

Figure 4: Emittance Profile in the CCDTL
Table 2: Tolerances of the CCDTL

- Field Amplitude : 1\%

- Field Gradient : $1 \%$

- Phase : 1 Degree

- Quadrupole displacement : 0.05mm

- Quadrupole rotation : 1 Degree

- Quadrupole strength (GL) : 1\%

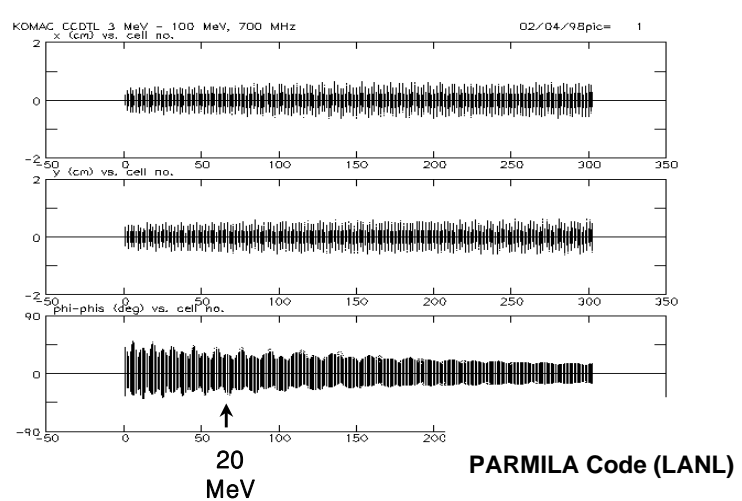

Figure 5: Beam trajectory with the errors in the CCDTL structure

\subsection{Mechanical Design}

To evaluate the manufacturing tolerances of the CCDTL cavity, the perturbation analysis of coupled resonators is used [4]. The tolerance of the cavity frequency is $100 \mathrm{kHz}$, and the coupling coefficient is 0.02 with a tolerance of $1 \%$.

The coupling coefficient between the accelerating cavity and the coupling cavity has been calculated by using the frequency shift between modes, which can be calculated with MAFIA code as shown in Fig. 6. Also, the machining tolerance for the coupling coefficient is $0.1 \mathrm{~mm}$, which can calculated by MAFIA code.

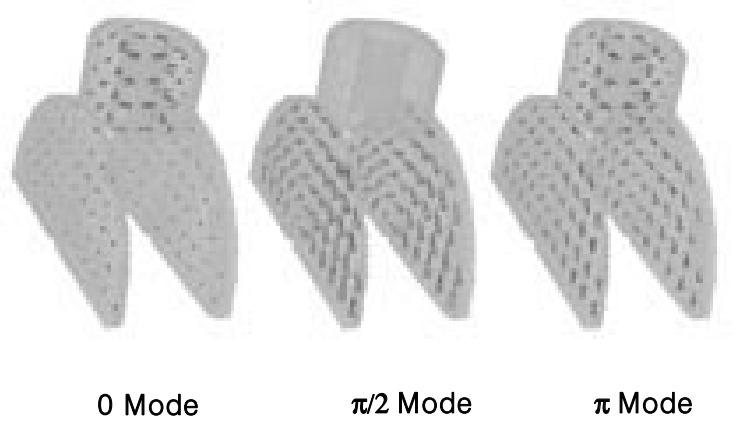

Figure 6: Modes of CCDTL

From this analysis, it has been found that there is no critical problem in fabricating the KOMAC CCDTL 
cavities. The technology for conventional CCL, which is well established, can be used in fabrication, tuning, installation, alignment, and operation.

For the cooling of CCDTL cavities, the water-cooling channel has been designed and analyzed by ANSYS code as shown in Fig. 7 [5]. The frequency shift due to the thermal expansion is $50 \mathrm{kHz}$. The coolant water velocity is $3 \mathrm{~m} / \mathrm{s}$, and the bulk temperature increase of the coolant water is less than $5 \mathrm{~K}$.
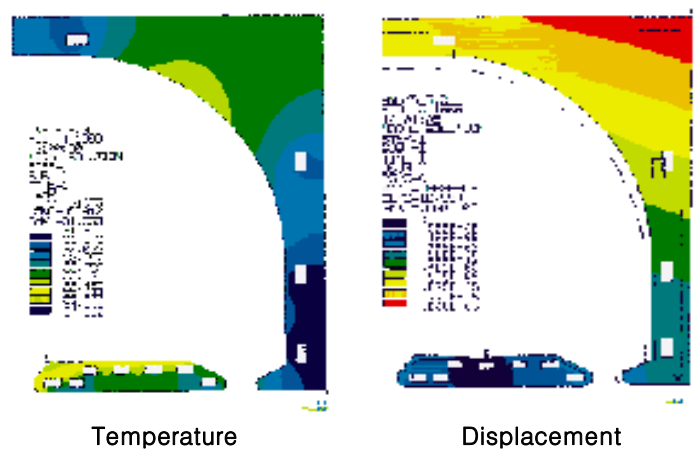

Figure 7: Thermal Analysis of CCDTL Cavity

\subsection{Cold Model}

The CCDTL cold models are fabricated to check the design, the tuning method, and the coupling coefficients and to check the engineering design. The Al cold model for design check is shown in Fig. 8. The test with this cold model is under study. The $\mathrm{Cu}$ cold model for fabrication check is shown in Fig. 9. The measured Q value of the cavity without brazing is $87 \%$ of the SUPERFISH calculated Q. The super-drilled coolant path is well fabricated, and this type cooling method will be used for the CCDTL construction. The brazing test will be performed.

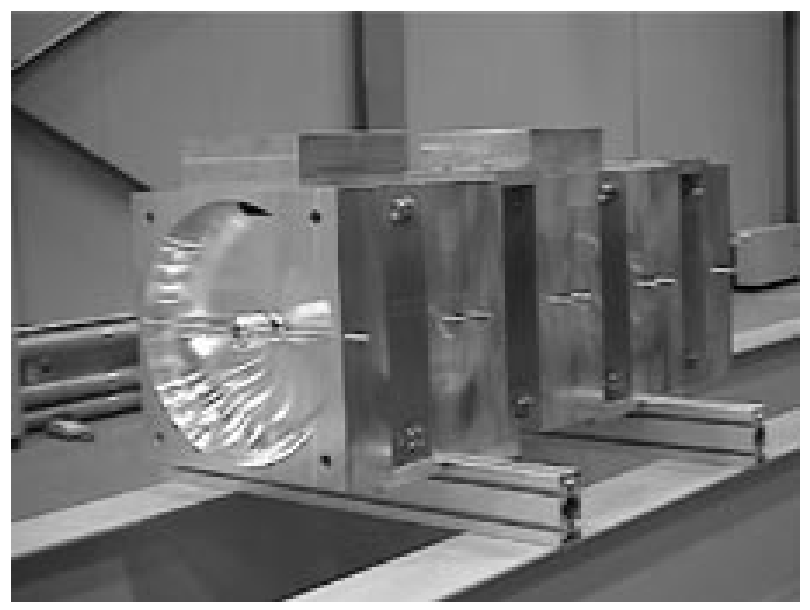

Figure 8: KOMAC CCDTL Cold Model at $20 \mathrm{MeV}$

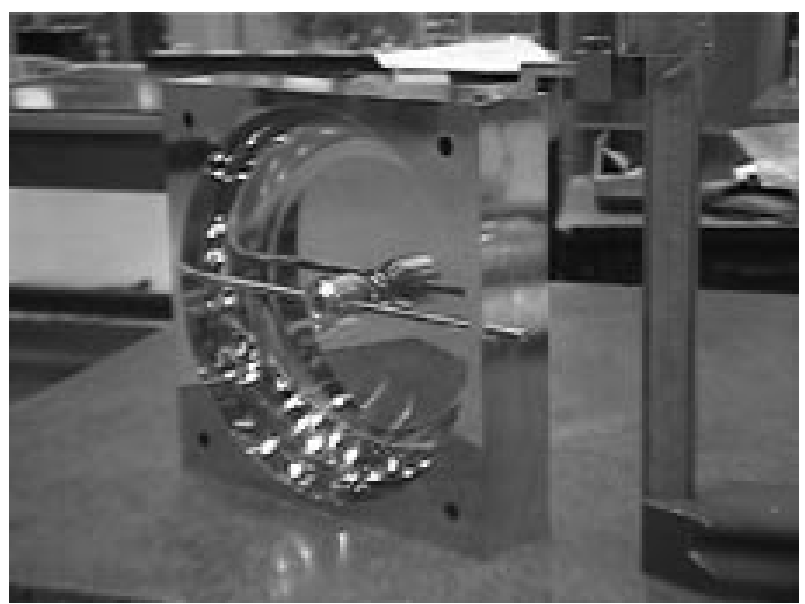

Figure 9: KOMAC CCDTL Cold Model at $20 \mathrm{MeV}$

\section{SUMMARY}

The design of KOMAC CCDTL is summarised in Table 3. The cold models are fabricated and the test is being performed. This model test will improve the KOMAC CCDTL design.

Table 3: Summary of KOMAC CCDTL

\begin{tabular}{|c|c|c|}
\hline Energy $(\mathrm{MeV})$ & $3 \sim 20$ & $20 \sim 100$ \\
\hline Current $(\mathrm{mA})$ & \multicolumn{2}{|c|}{$20 \mathrm{~mA}$} \\
\hline Structure & \multicolumn{2}{|c|}{ CCDTL } \\
\hline Focusing & \multicolumn{2}{|c|}{$8 \beta \lambda$ FODO } \\
\hline $\begin{array}{c}\text { Gaps per } \\
\text { Focusing Period }\end{array}$ & $4 \& 6$ & 8 \\
\hline Length $(\mathrm{m})$ & 29.8 & 94.2 \\
\hline \# of EMQ & 130 & 173 \\
\hline $\begin{array}{c}\text { \# of cavity } \\
\text { modules }\end{array}$ & 10 & 32 \\
\hline $\mathrm{P}_{\mathrm{cu}} / \mathrm{P}_{\text {total }}(\mathrm{MW})$ & $1.15 / 1.49$ & $3.43 / 5.03$ \\
\hline
\end{tabular}

\section{REFERENCES}

[1] J.H.Billen, F.L.Krawczyk, R.L.Wood, and L.M.Young, 'New RF Structure for Intermediate-Velocity Particles', Proceedings Of the 1994 International Linac Conference, Vol. 1, p. 341.

[2] 'National Spallation Neutron Source, Conceptual Design Report', the NSNS Collaboration, May 1997.

[3] S.Nath, J.H.Billen, J.E.Stovall, H.Takeda, and L.M. Young, 'Frontend Physics Design of APT Linac', Proceedings of the 1997 Particle Accelerator Conference.

[4] D.E.Nagle, E.A.Knopp, and B.C.Knapp, 'A Coupled Resonator Model for Standing Wave Accelerator Tanks', Rev. Sci. Instr. 38, 1583 (1967).

[5] R.L.Wood, W.L.Clark, F.Martinez, and F.E. Sigler, 'Thermal/ Structural Design and Fabrication Development of High Power CCDTL and CCL Sturctures', Proceedings of the 1997 Particle Accelerator Conference. 